\title{
Some beneficial effect on root caries from use of higher concentration fluoride toothpaste (5000 ppm F)
}

\section{Abstracted from}

\section{Srinivasan M, Schimmel M, Riesen M, et al.}

High-fluoride toothpaste: a multicentre randomized controlled trial in adults. Community Dent Oral Epidemiol 2013; Dec 20. doi: 10.1111/cdoe.12090. [Epub ahead of print] PubMed PMID: 24354454.

Address for correspondence: Frauke Müller, Division of Gerodontology and Removable Prosthodontics, School of Dental Medicine, 19 Rue Barthélemy-Menn, Geneva 1205, Switzerland. E-mail: frauke.mueller@unige.ch

\section{Question: Is a high-fluoride toothpaste (5000 ppm F) effective for the management of root caries?}

Design Single-blind, multi-centre, parallel, randomised controlled trial. Intervention Adult patients (18-75 years) with 10 or more natural teeth, and one or more root caries lesion in a tooth not crowned or compromised were included. Patients were randomised to receive a high fluoride toothpaste, $1.1 \%$ sodium fluoride (5000 ppm F) or a regular toothpaste (1350 ppm F). Packaging was identical and standard brushes were also provided. Patients were strictly instructed to refrain from using toothpastes or toothbrushes other than the ones provided and restricted from using mouth rinses.

Outcome measure The primary outcome measure was changes in the surface structure of the root caries lesions after the intervention. This was graded using a surface hardness score (HS) scale: Level 1: hard; Level 2: hard to leathery; Level 3: leathery; Level 4: leathery with local softening; Level 5: soft. Scores were recorded at baseline, three and six months. Examiners were masked to the group assignment.

Results One hundred and thirty-five patients (test group: $\mathrm{n}=67$; control group: $\mathrm{n}=68$ ) with 318 identified root caries lesions were randomised. Five patients were lost to follow up, three in the test group and two in the control. The groups were similar at baseline. Overall there was no difference between the two groups for the overall HS (test group: $\mathrm{HS}=$ $3.4 \pm 0.61$; control group: $\mathrm{HS}=3.4 \pm 0.66 ; \mathrm{P}=0.8757$, unpaired t-test). However the ANOVA revealed significantly better HS for the test group than for the control groups ( $\mathrm{T} 1$ : test group: $\mathrm{HS}=2.9 \pm 0.67$; control group: $\mathrm{HS}=3.1 \pm 0.75 ; \mathrm{T} 2$ : test group: $\mathrm{HS}=2.4 \pm 0.81$; control group: $\mathrm{HS}=2.8 \pm 0.79 ; \mathrm{P}<0.0001)$.

Conclusions The application of a high-fluoride containing dentifrice (5000 ppm F) in adults, twice daily, significantly improves the surface hardness of otherwise untreated root caries lesions when compared with the use of regular fluoride containing (1350 ppm F) toothpastes. Acknowledgements The authors' institutions received per capita remunerations and the products used in this clinical trial, from Colgate - Palmolive Company. Roger P. Ellwood and Michael Warncke are employees of the Colgate - Palmolive Company.

\section{Commentary}

The proportion of older people in the population is growing and their inadequate plaque control procedures make root caries an increasingly common oral health problem. Soft tissue recession due to age, traumatic tooth brushing habits, periodontal disease or periodontal treatment will unavoidably result in more tooth root surfaces that are at risk for the development of root caries. Restorative treatment of root caries is notoriously difficult. Post-treatment pain and hypersensitivity are very common, and this may contribute to increased tooth loss in many people with root caries.

A systematic review examining the effectiveness of fluoride in preventing dental caries in adults found fluoride (self- and professionally applied or water fluoridation) annually averted 0.29 carious coronal and 0.22 carious root surfaces, and concluded fluoride is effective among adults of all ages. ${ }^{1}$ A systematic review of preventive interventions for root caries also showed that additional fluoride appears to be a preventive and therapeutic treatment for root caries. ${ }^{2}$ A recent randomised controlled trial (RCT) in elderly disabled nursing home residents in Denmark has found that 5000 ppm F toothpaste is significantly more effective for controlling root caries lesion progression and promoting remineralisation compared to 1450 ppm F toothpaste. ${ }^{3}$

The aim of this RCT was to evaluate the effectiveness of highfluoride toothpaste (5000 ppm F) on root caries lesions in adults and to test the hypothesis that high concentration fluoride toothpaste would effectively improve the surface hardness in root caries lesions in adult patients. The results of this study do suggest a statistical benefit at both three and six months from the use of 5000 ppm F toothpaste. Although the methodology of this RCT is robust, there were a few limitations as highlighted by the authors in their discussion. It was not possible to produce identical packages for the test and control toothpaste. Consequently, the patients were aware of their group assignment although the outcome assessors were blind. Another shortcoming was the failure to recruit the sample size of 90 per group proposed by the power calculation. The power was not adequate to demonstrate that the high-fluoride group improved surface hardness better than the lower-fluoride group over time.

While reporting of this RCT follows closely the CONSORT statement (www.consort-statement.org), there was no mention of any harms or unintended effects in each group. It would be helpful to state the absence of any adverse events even if none occurred. This is particularly important, as high-fluoride toothpaste is a prescription only medicine. Many patients who require the use of high-fluoride 
toothpaste may have special needs and find spitting out the toothpaste difficult. This may result in chronic ingestion of toothpaste and lead to fluoride toxicity. ${ }^{4}$

As with most trials, the participants recruited were a highly selective group, and/so the generalisability of the findings of this study is not certain. While we have strong evidence of effectiveness of fluoride toothpaste in children and adolescents, ${ }^{5}$ only a small number of studies have been conducted in adults. While this study suggests a benefit from the use of the higher concentration fluoride toothpaste in adults in the short term, more information is required to better inform our adult patients on their use. The extra cost of prescription toothpaste can be a hurdle for some patients.

\section{Practice points}

- Dental practitioners should consider prescribing higher concentration fluoride toothpaste (5000 ppm F) for patients with root caries lesions

- Repeat prescriptions of high-fluoride toothpaste should not be dispensed without reassessing the patient.
C Albert Yeung

Department of Public Health, NHS Lanarkshire, Bothwell, Scotland, UK

1. Griffin SO, Regnier E, Griffin PM, Huntley V. Effectiveness of fluoride in preventing caries in adults. / Dent Res 2007; 86: 410-415.

2. Heijnsbroek M, Paraskevas S, Van der Weijden GA. Fluoride interventions for root caries: a review. Oral Health Prev Dent 2007; 5: 145-152.

3. Ekstrand KR, Poulsen JE, Hede B, Twetman S, Qvist V, Ellwood RP. A randomized clinical trial of the anti-caries efficacy of 5,000 compared to 1,450 ppm fluoridated toothpaste on root caries lesions in elderly disabled nursing home residents. Caries Res 2013; 47: 391-398.

4. North West Medicines Information Centre. Fluoride toothpaste - what are the dangers of chronic ingestion in adults? December 2011. http://www.evidence. nhs.uk/search?q=\%22Fluoride+toothpaste+-+what+are+the+ angers+of+ chronic+ingestion+in+adults $\% 22$

5. Walsh T, Worthington HV, Glenny AM, Appelbe P, Marinho VC, Shi X. Fluoride toothpastes of different concentrations for preventing dental caries in children and adolescents. Cochrane Database Syst Rev 2010; Issue 1. Art. No.: CD007868.

Evidence-Based Dentistry (2014), 15, 8-9. doi: 10.1038/sj.ebd.6400981 